\title{
Gev-Scale Accelerators Driven by Plasma-Modulated Pulses from Kilohertz Lasers
}

\author{
O. Jakobsson $\odot$, S. M. Hooker®, and R. Walczak®* \\ John Adams Institute for Accelerator Science and Department of Physics, University of Oxford, \\ Denys Wilkinson Building, Keble Road, Oxford OX1 3RH, United Kingdom
}

(Received 17 June 2021; revised 24 August 2021; accepted 28 September 2021; published 26 October 2021)

\begin{abstract}
We describe a new approach for driving GeV-scale plasma accelerators with long laser pulses. We show that the temporal phase of a long, high-energy driving laser pulse can be modulated periodically by copropagating it with a low-amplitude plasma wave driven by a short, low-energy seed pulse. Compression of the modulated driver by a dispersive optic generates a train of short pulses suitable for resonantly driving a plasma accelerator. Modulation of the driver occurs via well-controlled linear processes, as confirmed by good agreement between particle-in-cell (PIC) simulations and an analytic model. PIC simulations demonstrate that a $1.7 \mathrm{~J}, 1 \mathrm{ps}$ driver, and a $140 \mathrm{~mJ}, 40 \mathrm{fs}$ seed pulse can accelerate electrons to energies of $0.65 \mathrm{GeV}$ in a plasma channel with an axial density of $2.5 \times 10^{17} \mathrm{~cm}^{-3}$. This work opens a route to high repetition-rate, $\mathrm{GeV}$-scale plasma accelerators driven by thin-disk lasers, which can provide joule-scale, picosecond-duration laser pulses at multikilohertz repetition rates and high wall-plug efficiencies.
\end{abstract}

DOI: $10.1103 /$ PhysRevLett.127.184801

The acceleration fields generated in a laser-plasma accelerator (LPA) are of the order of the wave-breaking field $E_{\mathrm{wb}}=m_{e} c \omega_{p} / e$, and for parameters of interest [1] can reach $100 \mathrm{GV} \mathrm{m}^{-1}$-around 3 orders of magnitude greater than possible in radio-frequency accelerators. The duration of the particle bunches accelerated by a LPA are a fraction of the plasma period $T_{p}=2 \pi / \omega_{p}$, i.e., a few femtoseconds [2-8]. Here, $\omega_{p}=\left(n_{e} e^{2} / m_{e} \epsilon_{0}\right)^{1 / 2}$ is the plasma frequency, where $n_{e}$ is the electron density.

The development of LPAs has seen remarkable progress in recent years [9-11]. Micrometer-sized electron bunches with $\mathrm{GeV}$-scale energies can be generated from LPA stages a few centimeters long [12-16], and hence LPAs have the potential to drive highly compact sources of high-energy, femtosecond-duration particles and radiation [17-23]. Indeed, their proof-of-principle applications to x-ray imaging [24-26], photochemistry [27], and electron radiotherapy [28-30] have been demonstrated, and conceptual designs for LPA facilities have been developed [31,32].

Efficient excitation of the plasma wave requires that the duration of the driving laser pulse satisfies $\tau<T_{p} / 2$, and the peak laser intensity is of order $10^{18} \mathrm{~W} \mathrm{~cm}^{-2}$. Few laser systems can meet these challenging requirements, and in practice almost all LPAs are driven by Ti:sapphire laser pulses with $\tau \approx 40$ fs and peak powers up to the PW range. These lasers are limited [33] to pulse repetition rates $f_{\text {rep }} \lesssim$ $10 \mathrm{~Hz}$ and mean powers $\lesssim 100 \mathrm{~W}$, whereas many applications of LPAs would require operation at $f_{\text {rep }} \gtrsim 1 \mathrm{kHz}$, with mean laser powers in the $\mathrm{kW}$ range. To date, $\mathrm{kHz}$ operation of LPAs [34-39] has been limited to generating electron energies below $15 \mathrm{MeV}$ by the low pulse energies available from $\mathrm{kHz}$ Ti:sapphire lasers.
A further consideration of vital importance for future high-mean-power LPAs is the wall-plug efficiency. For Ti: sapphire lasers this is $<0.1 \%$ [40], and with laser-to-wake and wake-to-bunch efficiencies each less than 50\% [41], the overall efficiency is far from the $>10 \%$ required by future, cost-competitive high-energy LPAs [42].

Substantially more efficient lasers have been developed in recent years. For example, thin-disk lasers have opticalto-optical efficiencies exceeding 50\% and have recently generated pulse energies of $\sim 1 \mathrm{~J}$ at $f_{\text {rep }}=1 \mathrm{kHz}$ [43-45]. However, their long (picosecond) pulse duration makes them unsuitable for driving LPAs directly. Spectral broadening in gases has been shown [46-48] to reduce the duration of thin-disk laser pulses, but sub-100 fs pulses with the energy required to drive high-energy LPAs have yet to be demonstrated.

Many approaches for driving LPAs with long $\left(\tau>T_{p}\right)$ pulses have been investigated. In the plasma beat-wave accelerator (PBWA) [49] two long pulses of angular frequencies $\omega_{1}$ and $\omega_{2}=\omega_{1}+\omega_{p}$ are combined to create a modulated pulse which can resonantly excite a plasma wave. However, current high-efficiency laser technologies cannot generate a suitable second wavelength with the required high average power. Plasma waves can also be driven via self-modulation of long laser pulses [50,51], but this relies on stochastic, and highly nonlinear, processes which would make controlled injection and acceleration difficult.

In this Letter we describe a new approach which could be used to drive GeV-scale, multi-kHz LPAs with single, joulelevel, picosecond-duration pulses of the type recently demonstrated by thin-disk lasers. We use a one-dimensional (1D) analytic model and particle-in-cell (PIC) simulations to 


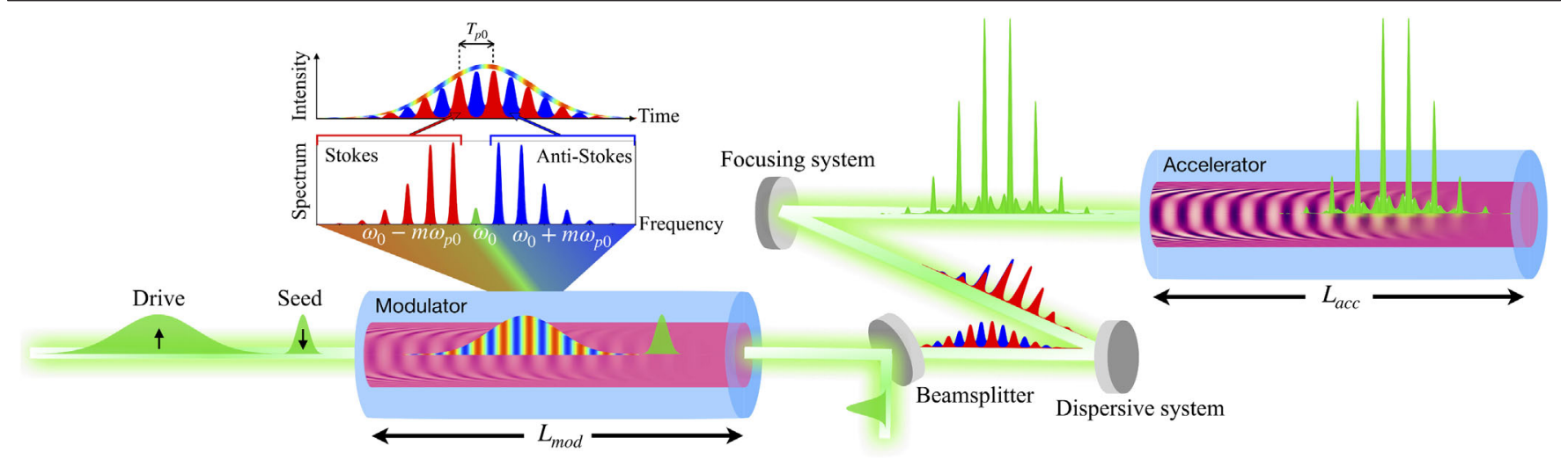

FIG. 1. Schematic diagram of a LPA driven by plasma-modulated laser pulses. A long, high-energy, drive laser pulse is phase modulated in the modulator stage by its interaction with the plasma wave driven by a short, low-energy seed pulse. The modulation generates sidebands at $\omega_{0}+m \omega_{p 0}$, although the temporal intensity profile of the drive pulse remains smooth. After leaving the modulator stage, the seed pulse is removed by a polarizing beam splitter, and the drive pulse is passed through (or reflected from) a dispersive optical system which removes the relative spectral phase of the sidebands, to form a train of short pulses spaced by $T_{p 0}=2 \pi / \omega_{p 0}$. This pulse train is focused into an accelerator stage, which comprises a plasma channel with the same on-axis density as that of the modulator stage. The pulse train resonantly excites a strong plasma wave that can be used for particle acceleration.

demonstrate the physics underlying this scheme. To provide an example, we simulate the acceleration of externally injected electrons by a $1.7 \mathrm{~J}, 1$ ps drive laser pulse to a mean energy of $0.65 \mathrm{GeV}$.

As shown in Fig. 1, our approach has three stages. In the first, the modulator stage, a short $\left(\tau_{\text {seed }} \lesssim T_{p 0} / 2\right)$, low energy seed pulse and a long $\left(\tau_{\text {drive }} \gg T_{p 0}\right)$, high-energy driving laser pulse are focused into a plasma waveguide. Here $T_{p 0}$ refers to the plasma period on axis. The seed pulse drives a low amplitude plasma wave which periodically modulates the temporal phase of the drive pulse, and hence generates frequency sidebands at $\omega_{0}+m \omega_{p 0}$. If isolated, the red- $(m<0)$ and blueshifted $(m>0)$ sidebands would form a pair of temporal pulse trains separated by $T_{p 0} / 2$, but with both sets present the temporal profile of the drive pulse remains smooth.

In the second stage, after removal of the seed pulse, the spectrally modulated drive pulse is converted into a temporally modulated train of short pulses, separated by $T_{p 0}$, by introducing a shift of $T_{p 0} / 2$ between the red- and blueshifted trains. This can be achieved by introducing a dispersive optical system [52-54] with the correct group delay dispersion (GDD). We note that a similar technique was proposed for plasma-based pulse compression in PBWA through electromagnetic (EM) cascading $[55,56]$. A major advantage of our scheme is that only a single highenergy drive pulse is required, rather than two pulses with a frequency difference matched to $\sim \omega_{p 0}$.

In the third (accelerator) stage, the pulse train is focused into a second plasma waveguide with the same axial density as the modulator, which resonantly excites a large-amplitude plasma wave for acceleration of externally injected electrons.

Considerable insight into these processes can be gained with the aid of a 1D analytic model (see Supplemental
Material [57]). The drive pulse has a normalized vector potential, $a(z, t)=b(z, t) \exp \left[\mathrm{i}\left(k_{0} z-\omega_{0} t\right)\right]$, where $\omega_{0}$ is the center frequency of the pulse, and $z$ is the coordinate along the propagation axis. After copropagating a distance $z$ with a linear plasma wave of density $n_{e}(z, t)=n_{0}+$ $\delta n \cos \left(k_{p 0} z-\omega_{p 0} t+\Delta \phi\right)$, the envelope of the pulse becomes [59],

$b(\zeta, \tau) \approx|b(\zeta, 0)| \sum_{m=-\infty}^{\infty} \mathrm{i}^{m} J_{m}(-\beta) \exp \left[\mathrm{im}\left(\omega_{p 0} \tau+\Delta \phi^{\prime}\right)\right]$

Here, $\zeta=z-v_{g} t$, where $v_{g}$ is the group velocity of the driver, $\quad \tau=t, \quad \beta=(1 / 2)\left(\omega_{p 0}^{2} / \omega_{0}\right)\left(\delta n / n_{0}\right)\left(z / v_{g}\right)$, and $\Delta \phi^{\prime}=-\left(\Delta \phi+\omega_{p 0} z / v_{p}\right)$, where $v_{p}$ is the phase velocity of the plasma wave. The generation of sidebands at $\omega_{0}+$ $m \omega_{p 0}$ is immediately apparent. In the temporal domain, the red- and blueshifted sidebands each form trains of pulses separated by $T_{p 0}$, with a relative shift [57] between the two trains of $T_{p 0} / 2$.

To demonstrate this scheme, and to gain further insight, we performed high-resolution 2D PIC simulations with the EPOCH code [60]. We compared these results with those obtained with a different PIC code implemented with cylindrical symmetry to check the validity of the $2 \mathrm{D}$ simulations [57]. The parameters of the drive and seed laser pulses were chosen to be similar to those recently demonstrated for thin-disk lasers operating at $f_{\text {rep }} \gtrsim 1 \mathrm{kHz}$ at a wavelength of $\lambda_{0}=1030 \mathrm{~nm}$. Both pulses were assumed to be bi-Gaussian, with a drive (seed) energy of $E_{p}^{\text {drive }}=600 \mathrm{~mJ}\left(E_{p}^{\text {seed }}=50 \mathrm{~mJ}\right)$ and a full width at half maximum pulse duration of $\tau_{\text {drive }}=1 \mathrm{ps}\left(\tau_{\text {seed }}=40 \mathrm{fs}\right)$. The pulses were separated by $1.7 \mathrm{ps}$. 

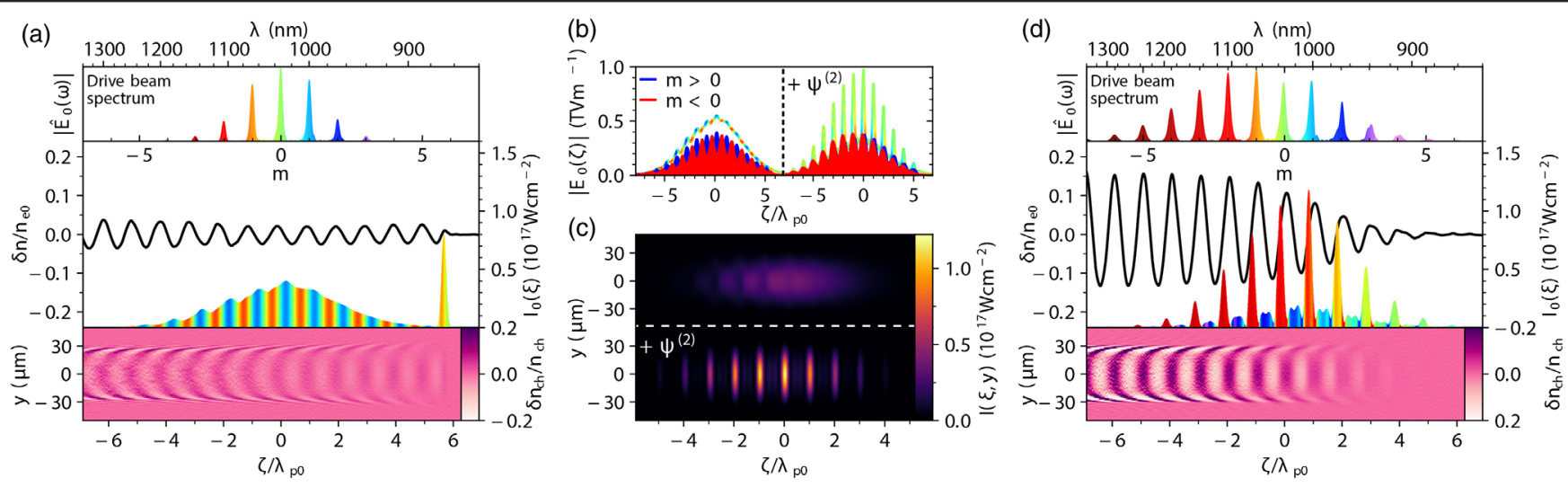

FIG. 2. Particle-in-cell simulations. (a) PIC simulations of the modulator stage, with results shown for the end of the modulator $(z=120 \mathrm{~mm})$. The top panel shows the on-axis spectral intensity of the drive pulse, plotted against a frequency scale $m=\left(\omega-\omega_{0}\right) / \omega_{p 0}$. The middle panel shows the longitudinal intensity profiles of the seed and driver pulses and the relative amplitude of the plasma wave on axis $(y=0)$. The bottom panel shows, for $z=120 \mathrm{~mm}$, a $2 \mathrm{D}$ plot of the electron density relative to the channel profile $\delta n_{\mathrm{ch}} / n_{\mathrm{ch}}=\left(n_{e}-n_{\mathrm{ch}}\right) / n_{\mathrm{ch}}$. The shading of the longitudinal profiles indicates the local effective frequency $-d \phi / d t$, where $\phi$ is the temporal phase, using the same color scale as the top panel. (b) The modulus of the on-axis electric field of the drive pulse together with the red- and blueshifted components before and after application of a quadratic spectral phase $\psi^{(2)}=-1480 \mathrm{fs}^{2}$. (c) The corresponding 2D intensity profiles. (d) The same plots as in (a) but at a distance $z=50 \mathrm{~mm}$ into the accelerator stage.

The plasma parameters of the modulator and accelerator stages were identical. For radii $r<r_{0}=1.2 w_{M}$, the electron density was $n_{c h}(r)=n_{e 0}+\left(1 / \pi r_{e} w_{M}^{2}\right)\left(r / w_{M}\right)^{\alpha}$, where $n_{e 0} \equiv n_{e}(0)=2.5 \times 10^{17} \mathrm{~cm}^{-3}$, and $w_{M}=30 \mu \mathrm{m}$ is approximately the spot size of the lowest-order channel mode. For $r>1.2 w_{M}, n_{e}(r)=n_{e}\left(r_{0}\right)$ for $\Delta r=10 \mu \mathrm{m}$, before decreasing linearly to zero in the same distance. The parameter $\alpha=10$, and hence the channels were steep sided, with finite losses.

The drive and seed pulses were focused to a spot size $w_{0}=w_{M}=30 \mu \mathrm{m}$ at the entrance to the modulator channel. Figure 2(a) shows that the seed pulse drives a plasma wave of amplitude $\delta n / n_{e 0} \approx 2 \%$ on axis, which generates sidebands on the drive pulse at $\omega_{0}+m \omega_{p 0}$, although its temporal profile remains smooth. Figures 2(b),2(c) show, after the modulator, the on-axis electric field (b) and temporal intensity profile (c) of the drive pulse, before and after the application of a quadratic spectral phase with a group delay dispersion (GDD) of $\psi^{(2)}=-1480 \mathrm{fs}^{2}$. This value of the GDD was determined numerically to produce the highest-intensity pulse train, and is in excellent agreement with the value $\psi_{\mathrm{opt}}^{(2)}= \pm 1485 \mathrm{fs}^{2}$ predicted by the $1 \mathrm{D}$ model [57]. It is evident that the dispersion converts the phase modulated, but temporally smooth, drive pulse into a train of short pulses separated by $T_{p 0}=220 \mathrm{fs}$. The use of seed and drive pulses of different wavelengths is explored in Ref. [57].

Figure 2(d) shows the drive pulse train, and the plasma wake, after $50 \mathrm{~mm}$ propagation in the accelerator stage. The pulse train resonantly excites a strong plasma wave with $\delta n / n_{e} \approx 15 \%$ over the whole length of the accelerator. The spectrum of the driver is further modulated by this plasma wave, leading to the formation of additional sidebands through EM cascading [56]; it is also redshifted, the shift increasing towards the back of the pulse train, where the wake amplitude is higher.

The results of the PIC simulation can be compared with the 1D model of the modulator stage [57]. Figure 3(a) shows, as a function of the length $L_{\text {mod }}$ of the modulator, the relative energies in the central, redshifted, and blueshifted bands of the drive pulse. It can be seen that the analytic model and PIC simulations are in close agreement. The main difference is that leakage in the plasma channel, which is not included in the 1D model, attenuates the drive pulse in the PIC simulation. Figures 3(b),3(c) show (b) the properties of the pulse train which would be produced if the drive pulse at that point was compressed by introducing a GDD optimized to yield the highest-intensity pulse train; and (c) the peak accelerating field produced by injecting these trains into the accelerator stage. Examples of the pulse trains generated for three values of $L_{\text {mod }}$ are shown in the insets of Fig. 3(c). Again, very good agreement is obtained. The peak pulse intensity is seen to grow approximately linearly with $L_{\text {mod }}$; this is expected from the 1D model, since the spectral bandwidth increases as $L_{\text {mod }}$, and hence the duration of each compressed pulse will vary as $L_{\text {mod }}^{-1}$. It can be seen that trains of pulses as short as $15 \mathrm{fs}$ can be generated. Although the peak intensities of the pulses increase linearly with $L_{\text {mod }}$, the peak accelerating field does not increase significantly for $L_{\text {mod }} \gtrsim 100 \mathrm{~mm}$. This is expected since for single, short $\left(\tau_{\text {drive }} \ll T_{p 0}\right)$ drive pulses the wake amplitude depends only on the energy of the pulse [61], and for resonant pulse trains the wake amplitude depends only on the total energy of the train. The excellent agreement between the 1D analytic model and the PIC 


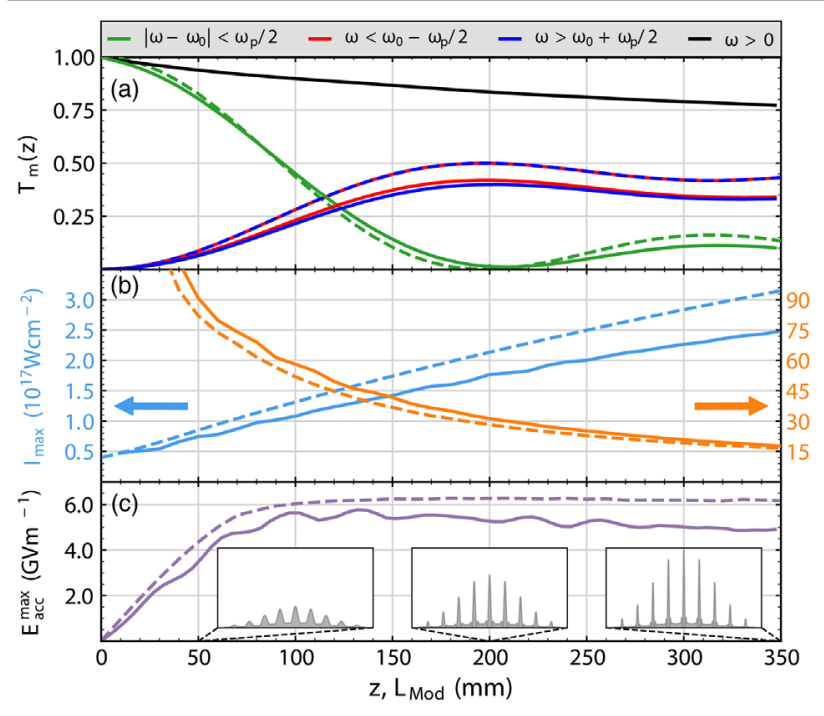

FIG. 3. Comparison of the results of the 1D analytic model (dashed lines) and PIC simulations (solid lines), plotted as a function of the length of the modulator stage. (a) The relative transmitted energies of the drive pulse (black), and of its components in the central band (i.e., $\left|\omega-\omega_{0}\right|<\omega_{p} / 2$, green), and in the blueshifted (i.e., $\left|\omega-\omega_{0}\right|>\omega_{p} / 2$, blue) and redshifted $\left(\left|\omega-\omega_{0}\right|<-\omega_{p} / 2\right.$, red) sidebands. (b) The peak intensity (blue), and FWHM duration (orange), of the most intense pulse in the train generated by applying a quadratic spectral phase, optimized to yield the highest-intensity pulse train, on the drive pulse emerging from the modulator stage. (c) The peak accelerating electric field produced by injecting into the accelerator stage the pulse trains which would be generated by compressing the drive pulse at that point in the modulator.

simulations demonstrates that, at least for this parameter range, the former captures the key physics involved in the operation of the modulator stage.

The analytic model and PIC simulation presented above demonstrate that plasma modulation can be used to convert a high-energy, long drive pulse to a train of short pulses suitable for resonant excitation of a large amplitude plasma wave. We emphasize that processes by which the drive pulse is modulated are linear, and can therefore be well controlled. Nevertheless it is important to avoid instabilities developing in either the modulator or accelerator stages. In this work the growth of instabilities, such as forward Raman scattering [62,63], was suppressed by operating in a favorable parameter regime, and by employing a plasma channel with small, but finite losses. These losses damp higher-order waveguide modes excited by the interaction between the drive pulse and the seed-driven plasma wave, preventing modulation of the temporal profile of the drive pulse, which would be susceptible to instabilitydriven growth [64].

In Fig. 4 we demonstrate that the scheme can be scaled to higher drive pulse energies, and hence higher acceleration gradients, whilst ensuring that the modulator operates in a well-controlled, linear regime. For these simulations the

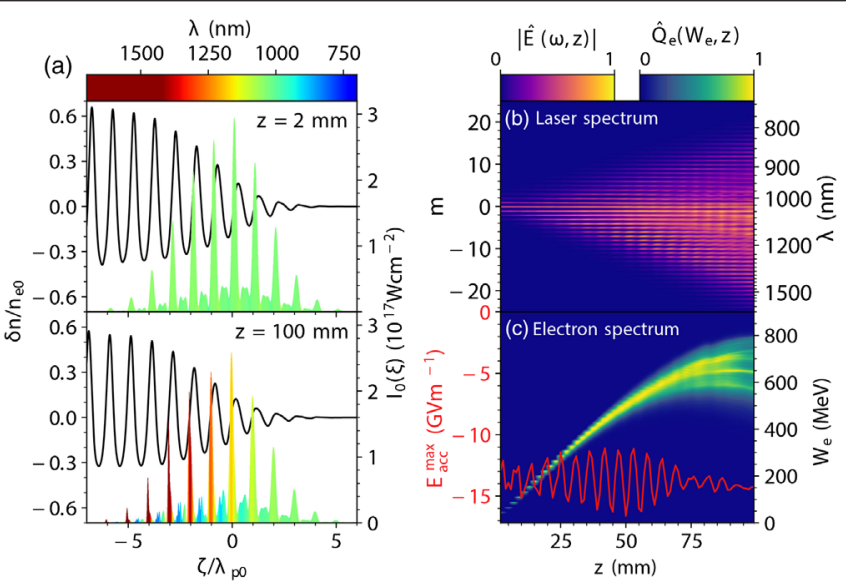

FIG. 4. Performance of a scaled accelerator with seed and drive pulse energies increased to $140 \mathrm{~mJ}$ and $1.7 \mathrm{~J}$, respectively. (a) The on-axis longitudinal profiles of the laser intensity and the relative electron density $\delta n / n_{0}$ at $z=2$ (top) and $z=100 \mathrm{~mm}$ (bottom) in the acceleration stage. The color scale shows the local laser wavelength. (b) Evolution of the normalized spectral intensity of the drive laser with propagation distance $z$ in the acceleration stage. (c) Evolution of the normalized energy spectrum $\hat{Q}_{e}\left(W_{e}, z\right)$ of the injected electron bunch with $z$.

parameters of the accelerator stage, and the axial density of the modulator stage, were the same as in Fig. 2. However, the matched spot size of the modulator was increased to $50 \mu \mathrm{m}$ in order to allow the seed and drive pulse energies to be increased to $140 \mathrm{~mJ}$ and $1.7 \mathrm{~J}$ while keeping their peak intensities the same as in Fig. 2. Figure 4(a) shows clearly that a quasilinear wakefield, with an amplitude considerably larger than that of Fig. 2, can be driven over the entire length of the $100 \mathrm{~mm}$ acceleration stage.

To demonstrate particle acceleration, a $1 \mathrm{pC}$ electron bunch of energy $35 \mathrm{MeV}, 5$ fs root-mean-square duration, and $4 \mu \mathrm{m}$ transverse width was injected into the focusing phase of the wakefield, at the position of peak acceleration. Figure 4(c) shows the evolution with $z$ of the normalized energy spectrum $\hat{Q}_{e}\left(W_{e}, z\right)$ of this bunch, where $W_{e}$ is the electron energy. It can be seen that the bunch maintains a relatively narrow energy spectrum up to $z \approx 50 \mathrm{~mm}$, at which point the mean energy is $\sim 500 \mathrm{MeV}$. At larger $z$, dephasing causes the energy spectrum to broaden. The mean electron energy at the end of the accelerator stage is $0.65 \mathrm{GeV}$. The laser-plasma energy transfer in the accelerator stage is found [57] to be $9 \%$.

The results of Fig. 4 show clearly that the concept described in this Letter can be scaled to generate higher particle energies without introducing unwanted instabilities in either modulator or acceleration stages. Our results serve to demonstrate the operation of this scheme, but further work will be required to fully explore its potential. For example, it is likely that the energy transfer efficiency could be increased, and the properties of the accelerated bunch improved, by optimizing the parameters of the drive laser 
and plasma channel, and the beam loading of the plasma wakefield.

In summary, we have presented a new approach for resonantly driving plasma accelerators with long laser pulses, based on copropagation with a low-amplitude plasma wave and compression by a dispersive optic of the spectrally modulated output to form a train of short pulses. This scheme was demonstrated via a 1D analytic model and 2D PIC simulations, which were found to be in excellent agreement. Numerical simulations showed that a quasilinear wakefield could be driven over a dephasing length to accelerate a test bunch to a mean energy $\sim 0.65 \mathrm{GeV}$.

We note that the key components required to realize this new approach have all recently been demonstrated, and, in principle, all are capable of multikilohertz operation. These include meter-scale, low-loss, all-optical plasma waveguides [65-71]; $100 \mathrm{~mJ}$-scale, sub-50 fs seed laser pulses [72]; and joule-level, few-picosecond, $1030 \mathrm{~nm}$ drive laser pulses $[43,45]$. The proposed scheme requires tight temporal and spatial overlap of multiple laser beams, the most challenging of which are (i) control of the delay between the electron bunch and the seed pulse ( $10 \mathrm{fs})$; and (ii) control of the pointing of the seed and drive pulses relative to the waveguide axes $(<10 \mu \mathrm{rad})$. These requirements have already been met $[68,69,71,73-76]$ in experiments operating at $f_{\text {rep }}=1 \mathrm{~Hz}$, and even tighter tolerances could be achieved with the improved feedback made possible by $\mathrm{kHz}$ operation.

The authors acknowledge useful discussions with A. Picksley and J. A. Holloway. This work was supported by the UK Science and Technology Facilities Council (STFC UK) [Grants No. ST/P002048/1 and No. ST/V001655/1]; the Engineering and Physical Sciences Research Council (EPSRC UK) [Grants No. EP/V006797/1 and No. EP/ R513295/1]. This material is based upon work supported by the Air Force Office of Scientific Research under Grant No. FA9550-18-1-7005. This work was supported by the European Union's Horizon 2020 research and innovation programme under Grant Agreement No. 653782. This work required significant computing resources which were funded by the plasma HEC Consortium [EPSRC Grant No. EP/R029149/1] and UKRI funding [ARCHER2 Pioneer Projects]. The development of the EPOCH code is supported in part by the UK EPSRC Grants No. EP/ G054950/1, No. EP/G056803/1, No. EP/G055165/1, and No. EP/M022463/1. Computing resources were provided by ARCHER and ARCHER2 [ARCHER2 PR17125] UK supercomputers as well as STFC Scientific Computing Department's SCARF cluster.

*roman.walczak@physics.ox.ac.uk

[1] T. Tajima and J. M. Dawson, Laser Electron Accelerator, Phys. Rev. Lett. 43, 267 (1979).
[2] J. Faure, C. Rechatin, A. Norlin, A. Lifschitz, Y. Glinec, and V. Malka, Controlled injection and acceleration of electrons in plasma wakefields by colliding laser pulses, Nature (London) 444, 737 (2006).

[3] J. van Tilborg, C. B. Schroeder, C. V. Filip, C. Tóth, C. G. R. Geddes, G. Fubiani, R. Huber, R. A. Kaindl, E. Esarey, and W. P. Leemans, Temporal Characterization of Femtosecond Laser-Plasma-Accelerated Electron Bunches Using Terahertz Radiation, Phys. Rev. Lett. 96, 014801 (2006).

[4] T. Ohkubo, A. Maekawa, R. Tsujii, T. Hosokai, K. Kinoshita, K. Kobayashi, M. Uesaka, A. Zhidkov, K. Nemoto, Y. Kondo, and Y. Shibata, Temporal characteristics of monoenergetic electron beams generated by the laser wakefield acceleration, Phys. Rev. ST Accel. Beams 10, 031301 (2007).

[5] A. D. Debus et al., Electron Bunch Length Measurements from Laser-Accelerated Electrons Using Single-Shot THz Time-Domain Interferometry, Phys. Rev. Lett. 104, 084802 (2010).

[6] O. Lundh, J. Lim, C. Rechatin, L. Ammoura, A. Ben-Ismaill, X. Davoine, G. Gallot, J. P. Goddet, E. Lefebvre, V. Malka, and J. Faure, Few femtosecond, few kiloampere electron bunch produced by a laser-plasma accelerator, Nat. Phys. 7, 219 (2011).

[7] A. Buck, J. Wenz, J. Xu, K. Khrennikov, K. Schmid, M. Heigoldt, J. M. Mikhailova, M. Geissler, B. Shen, F. Krausz, S. Karsch, and L. Veisz, Shock-Front Injector for HighQuality Laser-Plasma Acceleration, Phys. Rev. Lett. 110, 185006 (2013).

[8] M. Heigoldt, A. Popp, K. Khrennikov, J. Wenz, S.-W. Chou, S. Karsch, S. I. Bajlekov, S. M. Hooker, and B. Schmidt, Temporal evolution of longitudinal bunch profile in a laser wakefield accelerator, Phys. Rev. ST Accel. Beams 18, 121302 (2015).

[9] E. Esarey, C. Schroeder, and W. Leemans, Physics of laserdriven plasma-based electron accelerators, Rev. Mod. Phys. 81, 1229 (2009).

[10] S. M. Hooker, Developments in laser-driven plasma accelerators, Nat. Photonics 7, 775 (2013).

[11] C. Joshi, S. Corde, and W. B. Mori, Perspectives on the generation of electron beams from plasma-based accelerators and their near and long term applications, Phys. Plasmas 27, 070602 (2020).

[12] W. P. Leemans, B. Nagler, A. J. Gonsalves, C. Toth, K. Nakamura, C. G. R. Geddes, E. Esarey, C. B. Schroeder, and S. M. Hooker, GeV electron beams from a centimetre-scale accelerator, Nat. Phys. 2, 696 (2006).

[13] S. Kneip et al., Near-GeV Acceleration of Electrons by a Nonlinear Plasma Wave Driven by a Self-Guided Laser Pulse, Phys. Rev. Lett. 103, 035002 (2009).

[14] X. Wang et al., Quasi-monoenergetic laser-plasma acceleration of electrons to $2 \mathrm{GeV}$, Nat. Commun. 4, 1988 (2013).

[15] W. P. Leemans, A. J. Gonsalves, H. S. Mao, K. Nakamura, C. Benedetti, C. B. Schroeder, C. Toth, J. Daniels, D. E. Mittelberger, S. S. Bulanov, J. L. Vay, C. G. R. Geddes, and E. Esarey, Multi-GeV Electron Beams from CapillaryDischarge-Guided Subpetawatt Laser Pulses in the SelfTrapping Regime, Phys. Rev. Lett. 113, 245002 (2014). 
[16] A. J. Gonsalves et al., Petawatt Laser Guiding and Electron Beam Acceleration to $8 \mathrm{GeV}$ in a Laser-Heated Capillary Discharge Waveguide, Phys. Rev. Lett. 122, 084801 (2019).

[17] S. Kneip et al., Bright spatially coherent synchrotron x-rays from a table-top source, Nat. Phys. 6, 980 (2010).

[18] H.-P. Schlenvoigt, K. Haupt, A. Debus, F. Budde, O. Jackel, S. Pfotenhauer, H. Schwoerer, E. Rohwer, J. G. Gallacher, E. Brunetti, R. P. Shanks, S. M. Wiggins, and D. A. Jaroszynski, A compact synchrotron radiation source driven by a laser-plasma wakefield accelerator, Nat. Phys. 4, 130 (2008).

[19] M. Fuchs et al., Laser-driven soft-x-ray undulator source, Nat. Phys. 5, 826 (2009).

[20] K. T. Phuoc, S. Corde, C. Thaury, V. Malka, A. Tafzi, J.-P. Goddet, R. C. Shah, S. Sebban, and A. Rousse, All-optical compton gamma-ray source, Nat. Photonics 6, 308 (2012).

[21] S. Corde, K. Ta Phuoc, G. Lambert, R. Fitour, V. Malka, A. Rousse, A. Beck, and E. Lefebvre, Femtosecond x rays from laser-plasma accelerators, Rev. Mod. Phys. 85, 1 (2013).

[22] K. Khrennikov, J. Wenz, A. Buck, J. Xu, M. Heigoldt, L. Veisz, and S. Karsch, Tunable All-Optical Quasimonochromatic Thomson X-Ray Source in the Nonlinear Regime, Phys. Rev. Lett. 114, 195003 (2015).

[23] W. Wang, K. Feng, L. Ke, C. Yu, Y. Xu, R. Qi, Y. Chen, Z. Qin, Z. Zhang, M. Fang, J. Liu, K. Jiang, H. Wang, C. Wang, X. Yang, F. Wu, Y. Leng, J. Liu, R. Li, and Z. Xu, Free-electron lasing at 27 nanometres based on a laser wakefield accelerator, Nature (London) 595, 516 (2021).

[24] S. Kneip, C. McGuffey, F. Dollar, M. S. Bloom, V. Chvykov, G. Kalintchenko, K. Krushelnick, A. Maksimchuk, S. P. Mangles, T. Matsuoka, Z. Najmudin, C. A. Palmer, J. Schreiber, W. Schumaker, A. G. Thomas, and V. Yanovsky, X-ray phase contrast imaging of biological specimens with femtosecond pulses of betatron radiation from a compact laser plasma wakefield accelerator, Appl. Phys. Lett. 99, 093701 (2011).

[25] A. E. Hussein et al., Laser-wakefield accelerators for highresolution X-ray imaging of complex microstructures, Sci. Rep. 9, 3249 (2019).

[26] A. Ben-Ismail, O. Lundh, C. Rechatin, J. K. Lim, J. Faure, S. Corde, and V. Malka, Compact and high-quality gammaray source applied to $10 \mu \mathrm{m}$-range resolution radiography, Appl. Phys. Lett. 98, 264101 (2011).

[27] Y. A. Gauduel, Y. Glinec, J. P. Rousseau, F. Burgy, and V. Malka, High energy radiation femtochemistry of water molecules: Early electron-radical pairs processes, Eur. Phys. J. D 60, 121 (2010).

[28] L. Labate, D. Palla, D. Panetta, F. Avella, F. Baffigi, F. Brandi, F. Di Martino, L. Fulgentini, A. Giulietti, P. Köster, D. Terzani, P. Tomassini, C. Traino, and L. A. Gizzi, Toward an effective use of laser-driven very high energy electrons for radiotherapy: Feasibility assessment of multi-field and intensity modulation irradiation schemes, Sci. Rep. 10, 17307 (2020).

[29] R. Polanek, N. A. Hafz, Z. Lécz, D. Papp, C. Kamperidis, S. Brunner, E. R. Szabó, T. Tőkés, and K. Hideghéty, 1 kHz laser accelerated electron beam feasible for radiotherapy uses: A PIC-Monte Carlo based study, Nucl. Instrum. Methods Phys. Res., Sect. A 987, 164841 (2021).
[30] K. Svendsen, D. Guénot, J. B. Svensson, K. Petersson, A. Persson, and $\mathrm{O}$. Lundh, A focused very high energy electron beam for fractionated stereotactic radiotherapy, Sci. Rep. 11, 5844 (2021).

[31] ALEGRO Collaboration, Towards an advanced linear international collider, arXiv:1901.10370.

[32] The EuPRAXIA Consortium, EuPRAXIA conceptual design report, Eur. Phys. J. Special Topics 229, 3675 (2020).

[33] J. W. Dawson et al., High average power lasers for future particle accelerators, AIP Conf. Proc. 1507, 147 (2012).

[34] Z. H. He, B. Hou, J. A. Nees, J. H. Easter, J. Faure, K. Krushelnick, and A. G. Thomas, High repetition-rate wakefield electron source generated by few-millijoule, 30 fs laser pulses on a density downramp, New J. Phys. 15, 053016 (2013).

[35] D. Guénot, D. Gustas, A. Vernier, B. Beaurepaire, F. Böhle, M. Bocoum, M. Lozano, A. Jullien, R. Lopez-Martens, A. Lifschitz, and J. Faure, Relativistic electron beams driven by $\mathrm{kHz}$ single-cycle light pulses, Nat. Photonics 11, 293 (2017).

[36] F. Salehi, A. G. Goers, G. A. Hine, L. Feder, D. Kuk, B. Miao, D. Woodbury, K. Y. Kim, and H. M. Milchberg, MeV electron acceleration at $1 \mathrm{kHz}$ with $<10 \mathrm{~mJ}$ laser pulses, Opt. Lett. 42, 215 (2017).

[37] D. Gustas, D. Guénot, A. Vernier, S. Dutt, F. Böhle, R. Lopez-Martens, A. Lifschitz, and J. Faure, High-charge relativistic electron bunches from a $\mathrm{kHz}$ laser-plasma accelerator, Phys. Rev. Accel. Beams 21, 013401 (2018).

[38] L. Rovige, J. Huijts, I. Andriyash, A. Vernier, V. Tomkus, V. Girdauskas, G. Raciukaitis, J. Dudutis, V. Stankevic, P. Gecys, M. Ouille, Z. Cheng, R. Lopez-Martens, and J. Faure, Demonstration of stable long-term operation of a kilohertz laser-plasma accelerator, Phys. Rev. Accel. Beams 23, 093401 (2020).

[39] F. Salehi, M. Le, L. Railing, M. Kolesik, and H. M. Milchberg, Laser-Accelerated, Low-Divergence 15-MeV Quasimonoenergetic Electron Bunches at $1 \mathrm{kHz}$, Phys. Rev. X 11, 021055 (2021).

[40] B. Hidding, S. Hooker, S. Jamison, B. Muratori, C. Murphy, Z. Najmudin, R. Pattathil, G. Sarri, M. Streeter, C. Welsch, M. Wing, and G. Xia, Plasma Wakefield Accelerator Research 2019-2040, http://pwasc.org.uk/uk-roadmapdevelopment.

[41] M. J. Streeter et al., Characterisation of laser wakefield acceleration efficiency with octave spanning near-ir spectrum measurements, arXiv:2011.01370.

[42] F. Albert et al., 2020 roadmap on plasma accelerators, New J. Phys. 23, 031101 (2021).

[43] C. Herkommer, P. Krötz, R. Jung, S. Klingebiel, C. Wandt, R. Bessing, P. Walch, T. Produit, K. Michel, D. Bauer, R. Kienberger, and T. Metzger, Ultrafast thin-disk multipass amplifier with $720 \mathrm{~mJ}$ operating at kilohertz repetition rate for applications in atmospheric research, Opt. Express 28, 30164 (2020).

[44] T. Produit et al., The laser lightning rod project, Eur. Phys. J. Appl. Phys. 93, 10504 (2021).

[45] Y. Wang, H. Chi, C. Baumgarten, K. Dehne, A. R. Meadows, A. Davenport, G. Murray, A. B. Reagaan, 
C. S. Menoni, and J. J. Rocca, 1.1 J Yb:YAG picosecond laser at $1 \mathrm{kHz}$ repetition rate, Opt. Lett. 45, 6615 (2020).

[46] J. J. Rocca, B. E. Schmidt, H. Wang, Y. Wang, T. Tarkil, H. Chi, and C.S. Menoni, High average power femtosecond laser driver for plasma accelerators by compression of spectrally broadened high energy $\mathrm{Yb}$ :YAG laser pulses, in Snowmass 2021 AF7/AF6: Advanced Accelerator Concepts (2021), https://indico.fnal.gov/event/45651/ contributions/197898/attachments/134960/167168/LOI124-Rocca_et_al_CSU_Snowmass_2021_slides.pdf.

[47] G. Fan, P. A. Carpeggiani, Z. Tao, G. Coccia, R. Safaei, E. Kaksis, A. Pugzlys, F. Légaré, B. E. Schmidt, and A. Baltuška, $70 \mathrm{~mJ}$ nonlinear compression and scaling route for an $\mathrm{Yb}$ amplifier using large-core hollow fibers, Opt. Lett. 46, 896 (2021).

[48] M. Kaumanns, D. Kormin, T. Nubbemeyer, V. Pervak, and S. Karsch, Spectral broadening of $112 \mathrm{~mJ}, 1.3 \mathrm{ps}$ pulses at $5 \mathrm{kHz}$ in a $\mathrm{LG}_{10}$ multipass cell with compressibility to $37 \mathrm{fs}$, Opt. Lett. 46, 929 (2021).

[49] Y. Kitagawa, T. Matsumoto, T. Minamihata, K. Sawai, K. Matsuo, K. Mima, K. Nishihara, H. Azechi, K. A. Tanaka, H. Takabe, and S. Nakai, Beat-Wave Excitation of Plasma Wave and Observation of Accelerated Electrons, Phys. Rev. Lett. 68, 48 (1992).

[50] N. Andreev, L. Gorbunov, V. Kirsanov, A. Pogosova, and R. Ramazashvili, Resonant excitation of wakefields by a laser pulse in a plasma, JETP Lett. 55, 571 (1992).

[51] P. M. King, K. Miller, N. Lemos, J. L. Shaw, B. F. Kraus, M. Thibodeau, B. M. Hegelich, J. Hinojosa, P. Michel, C. Joshi, K. A. Marsh, W. Mori, A. Pak, A. G. Thomas, and F. Albert, Predominant contribution of direct laser acceleration to high-energy electron spectra in a low-density selfmodulated laser wakefield accelerator, Phys. Rev. Accel. Beams 24, 011302 (2021).

[52] S. Hädrich, J. Rothhardt, S. Demmler, M. Tschernajew, A. Hoffmann, M. Krebs, A. Liem, O. de Vries, M. Plötner, S. Fabian, T. Schreiber, J. Limpert, and A. Tünnermann, Scalability of components for $\mathrm{kW}$-level average power few-cycle lasers, Appl. Opt. 55, 1636 (2016).

[53] C. Grebing, M. Müller, J. Buldt, H. Stark, and J. Limpert, Kilowatt-average-power compression of millijoule pulses in a gas-filled multi-pass cell, Opt. Lett. 45, 6250 (2020).

[54] D. A. Alessi, H. T. Nguyen, J. A. Britten, P. A. Rosso, and C. Haefner, Low-dispersion low-loss dielectric gratings for efficient ultrafast laser pulse compression at high average powers, Opt. Laser Technol. 117, 239 (2019).

[55] S. Kalmykov and G. Shvets, Compression of Laser Radiation in Plasmas using Electromagnetic Cascading, Phys. Rev. Lett. 94, 235001 (2005).

[56] S. Kalmykov and G. Shvets, Compression of laser radiation in plasmas via electromagnetic cascading, Phys. Plasmas 13, 056707 (2006).

[57] See Supplemental Material at http://link.aps.org/ supplemental/10.1103/PhysRevLett.127.184801 for further details on the linear theory and particle-in-cell simulations presented in this letter, which includes Ref. [58].

[58] R. Lehe, M. Kirchen, I. A. Andriyash, B. B. Godfrey, and J.L. Vay, A spectral, quasi-cylindrical and dispersion-free Particle-In-Cell algorithm, Comput. Phys. Commun., 203, 66 (2016).
[59] E. Esarey, A. Ting, and P. Sprangle, Frequency shifts induced in laser pulses by plasma waves, Phys. Rev. A 42, 3526 (1990).

[60] T. D. Arber, K. Bennett, C. S. Brady, A. Lawrence-Douglas, M. G. Ramsay, N. J. Sircombe, P. Gillies, R. G. Evans, H. Schmitz, A. R. Bell, and C. P. Ridgers, Contemporary particle-in-cell approach to laser-plasma modelling, Plasma Phys. Controlled Fusion 57, 113001 (2015).

[61] F. Dorchies, F. Amiranoff, V. Malka, J. R. Marquès, A. Modena, D. Bernard, F. Jacquet, P. Miné, B. Cros, G. Matthieussent, P. Mora, A. Solodov, J. Morillo, and Z. Najmudin, Acceleration of injected electrons in a laser wakefield experiment, Phys. Plasmas 6, 2903 (1999).

[62] C. D. Decker, W. B. Mori, T. Katsouleas, and D. E. Hinkel, Spatial temporal theory of Raman forward scattering, Phys. Plasmas 3, 1360 (1996).

[63] W. B. Mori, The physics of the nonlinear optics of plasmas at relativistic intensities for short-pulse lasers, IEEE J. Quantum Electron. 33, 1942 (1997).

[64] T. M. Antonsen and P. Mora, Leaky Channel Stabilization of Intense Laser Pulses in Tenuous Plasmas, Phys. Rev. Lett. 74, 4440 (1995).

[65] R. J. Shalloo, C. Arran, L. Corner, J. Holloway, J. Jonnerby, R. Walczak, H. M. Milchberg, and S. M. Hooker, Hydrodynamic optical-field-ionized plasma channels, Phys. Rev. E 97, 053203 (2018).

[66] R. J. Shalloo, C. Arran, A. Picksley, A. von Boetticher, L. Corner, J. Holloway, G. Hine, J. Jonnerby, H. M. Milchberg, C. Thornton, R. Walczak, and S. M. Hooker, Low-density hydrodynamic optical-field-ionized plasma channels generated with an axicon lens, Phys. Rev. Accel. Beams 22, 041302 (2019).

[67] S. Smartsev, C. Caizergues, K. Oubrerie, J. Gautier, J.-P. Goddet, A. Tafzi, K. T. Phuoc, V. Malka, and C. Thaury, Axiparabola: a long-focal-depth, high-resolution mirror for broadband high-intensity lasers, Opt. Lett. 44, 3414 (2019).

[68] A. Picksley, A. Alejo, J. Cowley, N. Bourgeois, L. Corner, L. Feder, J. Holloway, H. Jones, J. Jonnerby, H. M. Milchberg, L. R. Reid, A. J. Ross, R. Walczak, and S. M. Hooker, Guiding of high-intensity laser pulses in 100-mmlong hydrodynamic optical-field-ionized plasma channels, Phys. Rev. Accel. Beams 23, 081303 (2020).

[69] A. Picksley, A. Alejo, R. J. Shallo, C. Arran, A. von Boetticher, L. Corner, J. A. Holloway, J. Jonnerby, O. Jakobsson, C. Thornton, R. Walczak, and S. M. Hooker, Meter-scale conditioned hydrodynamic optical-field-ionized plasma channels, Phys. Rev. E 102, 053201 (2020).

[70] L. Feder, B. Miao, J. E. Shrock, A. Goffin, and H. M. Milchberg, Self-waveguiding of relativistic laser pulses in neutral gas channels, Phys. Rev. Research 2, 043173 (2020).

[71] B. Miao, L. Feder, J. E. Shrock, A. Goffin, and H. M. Milchberg, Optical Guiding in Meter-Scale Plasma Waveguides, Phys. Rev. Lett. 125, 074801 (2020).

[72] Thales Laser Group, ALPHA kHz-High Repetition Rate Ti:Sa Laser Series, https://www.thalesgroup.com/en/laserdatasheets.

[73] G. Genoud, F. Wojda, M. Burza, A. Persson, and C. G. Wahlström, Active control of the pointing of a multiterawatt laser, Rev. Sci. Instrum. 82, 033102 (2011). 
[74] A. R. Maier, N. M. Delbos, T. Eichner, L. Hübner, S. Jalas, L. Jeppe, S. W. Jolly, M. Kirchen, V. Leroux, P. Messner, M. Schnepp, M. Trunk, P. A. Walker, C. Werle, and P. Winkler, Decoding Sources of Energy Variability in a Laser-Plasma Accelerator, Phys. Rev. X 10, 031039 (2020).

[75] S. Jalas, M. Kirchen, P. Messner, P. Winkler, L. Hübner, J. Dirkwinkel, M. Schnepp, R. Lehe, and A. R. Maier,
Bayesian Optimization of a Laser-Plasma Accelerator, Phys. Rev. Lett. 126, 104801 (2021).

[76] Y. Wu, J. Hua, Z. Zhou, J. Zhang, S. Liu, B. Peng, Y. Fang, X. Ning, Z. Nie, F. Li, C. Zhang, C.-H. Pai, Y. Du, W. Lu, W. B. Mori, and C. Joshi, High-throughput injectionacceleration of electron bunches from a linear accelerator to a laser wakefield accelerator, Nat. Phys. 17, 801 (2021). 